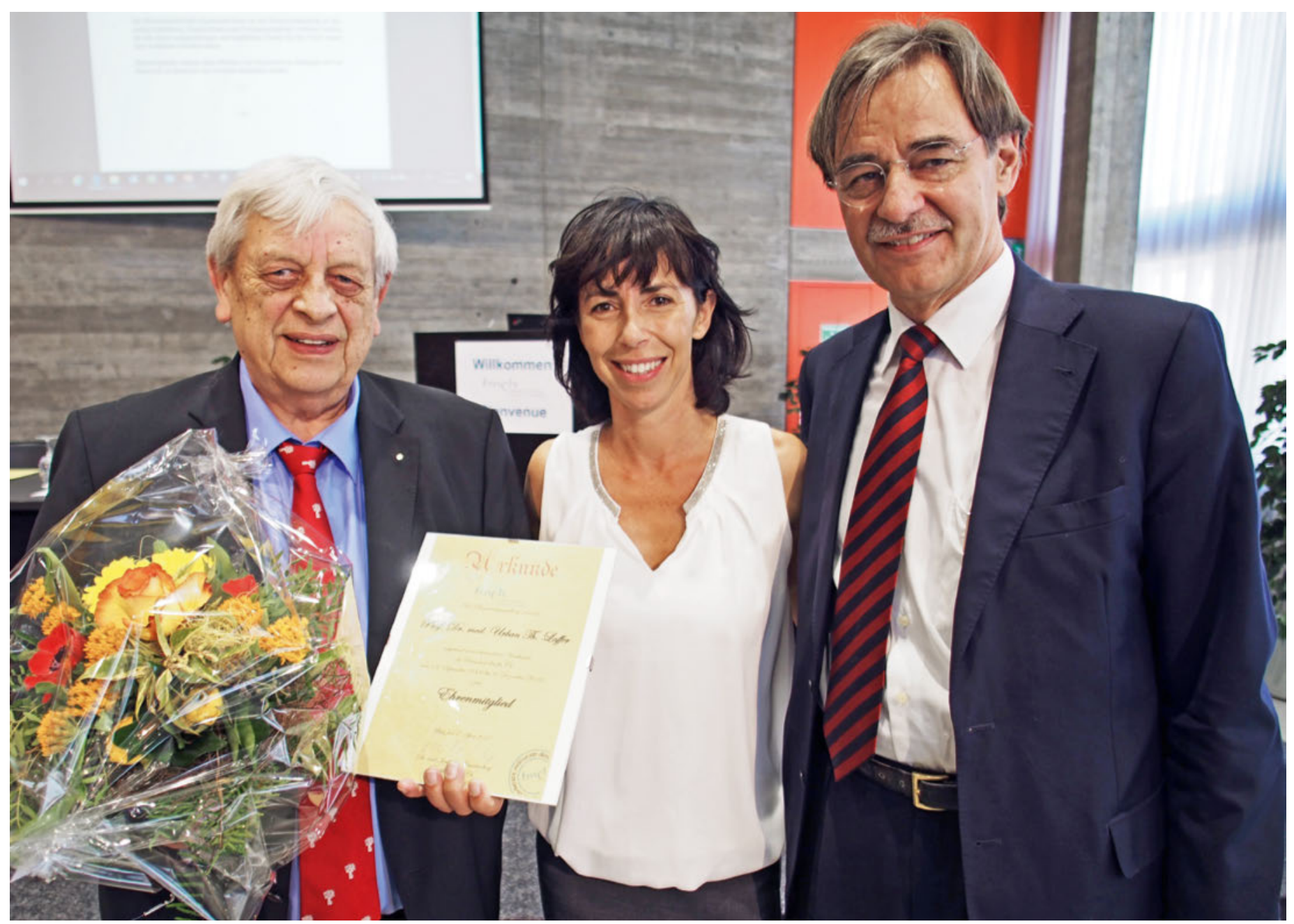

Der Geehrte, Urban Laffer, mit seinem Nachfolger Josef E. Brandenberg (rechts). Dame in der Mitte: Angeles Navarro von der Geschäftsstelle der fmCh.

\title{
Blumen für ein Urgestein
}

\section{Daniel Lüthi}

Freier Journalist und Fotograf, Medientrainer, Bern

Zwölf Jahre lang war er ihr prägender Präsident. Jetzt hat die fmCh, der Dachverband von rund zwanzig chirurgisch und invasiv tätigen Fachgesellschaften, Prof. Dr. med. Urban Laffer zu ihrem Ehrenmitglied ernannt.

Der Festakt fand in «seiner» Stadt statt: in Biel, wo Urban Laffer am Spitalzentrum von 1995 bis 2012 Chefarzt der chirurgischen Klinik und dann bis 2016 noch Medizinischer Leiter war. Die fmCh musste an ihrer Plenarversammlung im Bieler Kongresshaus zuerst die Statuten und das Geschäftsreglement ändern, bevor sie Urban Laffer - gestützt auf den neuen Punkt 5, «Ehrenmitgliedschaft ad personam» - nach vorne bit- ten konnte. Die führenden Exponenten des Verbandes überreichten ihm Urkunde und Blumenstrauss: sein Nachfolger Josef E. Brandenberg, Generalsekretär Markus Trutmann und Angeles Navarro von der Geschäftsstelle.

In einer sehr kurzen Dankesrede erhellte Urban Laffer einiges auch zwischen den Zeilen: "Zwölf Jahre sind eine lange Zeit», sagte er. «Ich kann nicht sagen, dass 
ich Sie nicht vermissen würde.» Und: Diese Auszeichnung freue ihn sehr. Dann ging die Versammlung sehr rasch zur Tagesordnung über: Bilanz und Erfolgsrechnung standen auf dem Programm. Der erste, der eine Frage stellte, war das eben gewählte, erste Ehrenmitglied - Urban Laffer.

Daniel Lüthi hat ihn nach seiner Ehrung zum Interview gebeten:

\section{Daniel Lüthi: Was bedeutet Ihnen diese Ehren- mitgliedschaft?}

Urban Laffer: Nach meinem Engagement zur Gründung der fmCh und nach zwölf Jahren als deren Präsident bedeutet mir diese Ehrung viel - sie berührt mich sehr.

Sie wurden für Ihre «besonderen Verdienste» geehrt was gehört aus Ihrer Sicht dazu?

Geschäftsleitung und Vorstand ist es gelungen, die fmCh als Verband zu festigen und neue Fachgesellschaften als Mitglieder zu gewinnen. Aus anfänglich 12 Fachgesellschaften sind es mittlerweile 19 geworden, bald werden es noch mehr sein. Damit ist die fmCh zu einem der grössten ärztlichen Verbände hierzulande gewachsen. Vor allem aber: Unsere Stimme wird nicht nur in der Ärzteschaft, sondern auch in der Öffentlichkeit und in der Politik gehört. Das kommt allen invasiv und chirurgisch tätigen Ärztinnen und Ärzten zugute.

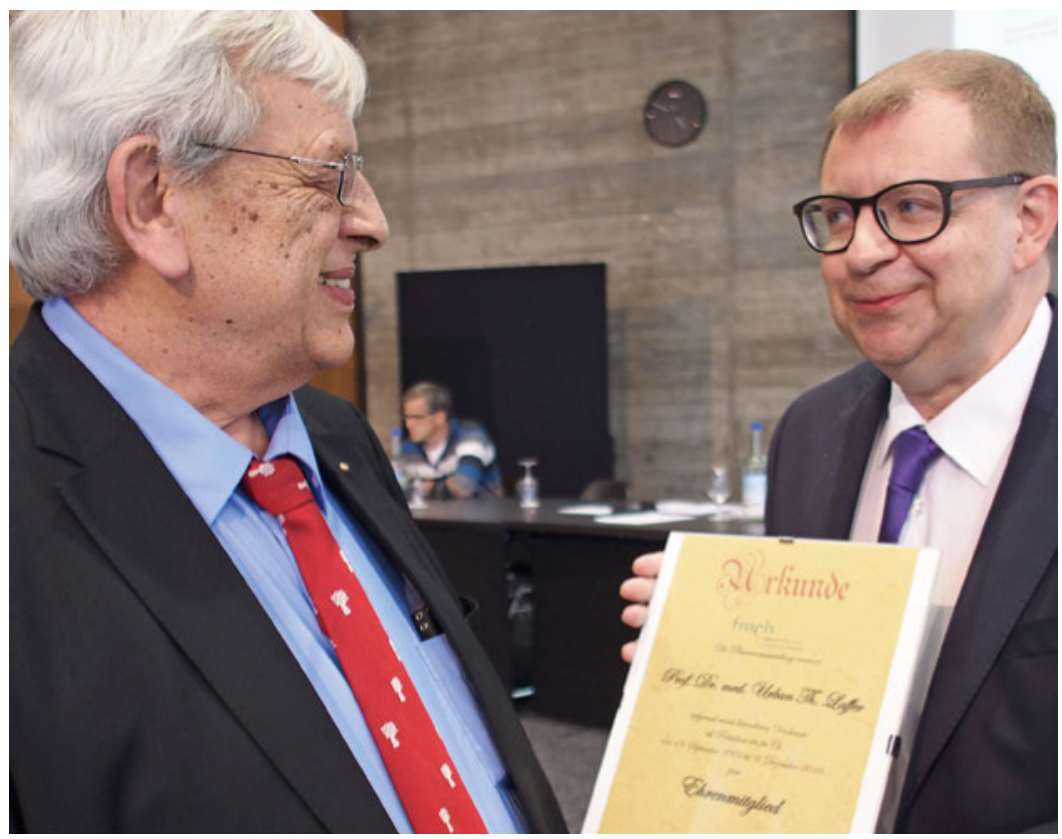

Markus Trutmann, Generalsekretär der fmCh, überreicht Ehrenmitglied Urban Laffer seine Urkunde.

\section{Was ist Ihnen nicht gelungen - was haben Sie an} wichtigen Aufgaben Ihrem Nachfolger übergeben?

Viele kleinere und grössere Aufgaben, die wir angepackt haben, haben wir nicht umsetzen können. Entweder sind wir an unseren finanziellen oder personellen Ressourcen gescheitert, oder aber am standespolitischen oder politischen Umfeld. Durch meinen Rücktritt musste ich notgedrungen die laufenden, schwierigen Arbeiten an der TARMED-Revision an meinen Nachfolger übergeben - eine Aufgabe, um die ich Josef Brandenberg wahrhaftig nicht beneide!

\section{Werden Sie sich in Ihrer neuen Rolle weiterhin zu Wort melden und aktiv sein?}

Ich hoffe sehr, dass meine neue Rolle als «Ehrenmitglied» nicht nur eine leere Worthülse sein wird, sondern dass ich mit der fmCh eng in Verbindung bleiben darf. Ich stehe mit Rat und Tat weiterhin sehr gerne zur Verfügung. Dies im Wissen, dass mein Nachfolger Josef Brandenberg die fmCh engagiert und motiviert und mit viel frischem Wind in eine gute Zukunft führen wird.

Wofür oder gegen wen oder was müssen die Chirurgen Ihrer Meinung nach künftig vor allem kämpfen? Zu kämpfen gilt es gegen ein Globalbudget und die Verstaatlichung der Medizin. Weniger Kampf, aber grosses Engagement ist gefragt für den guten Ruf und die Qualität unserer invasiven und chirurgischen Tätigkeiten und für die Sorge um den Nachwuchs unserer Fachgesellschaften.

\section{Wie geht es Ihnen ohne Ihre Ämter? Was vermissen Sie?}

Es wäre gelogen, zu behaupten, dass nach über 40 Jahren chirurgischer, spital- und standespolitischer Tätigkeit der Schritt in den "Ruhestand» einfach wäre. Ich vermisse noch vieles, ganz besonders den regelmässigen Kontakt und Gedankenaustausch mit Kolleginnen und Kollegen.

\section{Wo werden Sie die Urkunde, die Sie von der fmCh} erhalten haben, platzieren?

Die Urkunde hat in meinem Arbeitszimmer bereits einen würdigen Platz erhalten - neben anderen Ehrungen, die ich in den vergangenen Monaten und Jahren empfangen durfte.

\section{Bildnachweis}

Fotos Daniel Lüthi 